\title{
A cointegration-based monitoring method for rolling bearings working in time-varying operational conditions
}

\author{
Ali akbar Tabrizi ${ }^{1}$, Hussein Al-Bugharbee ${ }^{2}$, Irina Trendafilova², Luigi Garibaldi ${ }^{3}$ \\ 1) Manufacturing Group, IKID University of Applied Science and Technology, Iran \\ 2) DASM Group, Department of Mechanical and Aeronautical Engineering, University of Strathclyde, UK \\ 3) DIRG Group, Department of Mechanical and Aerospace Engineering, Politecnico di Torino, Italy
}

\section{Corresponding author:}

Ali akbar Tabrizi

Manufacturing Group, IKID University of Applied Science and Technology, 5th km Karaj- Qazvin freeway, Karaj, Iran

Email: a.tabrizi@IKID.IR

\begin{abstract}
Most conventional diagnostic methods for fault diagnosis in rolling bearings are able to work only for the case of stationary operating conditions (constant speed and load), whereas, bearings often work at time-varying conditions. Some methods have been proposed for damage detection in bearings working under time-varying speed conditions. However, their application might increase the instrumentation cost because of providing a phase reference signal. Furthermore, some methods such as order tracking methods can only be applied for limited speed variations.

In this study, a novel combined method for fault detection in rolling bearings based on cointegration is proposed for the development of fault features which are sensitive to the presence of defects while in the same time they
\end{abstract}


are insensitive to changes in the operational conditions. The method makes use solely of the measured vibration signals and does not require any additional measurements while it can identify defects even for considerable speed variations. The signals acquired during run-up condition are decomposed into zero-mean modes called intrinsic mode functions using the Performance Improved Ensemble Empirical Mode Decomposition method. Then, the cointegration, which is finding stationary linear combination of some non-stationary time series, is applied to the intrinsic mode functions to extract stationary residuals. The feature vectors are created by applying the Teager-Kaiser energy operator to the obtained stationary residuals. Finally, the feature vectors of the healthy bearing signals are utilized to construct a separating hyperplane using the one-class support vector machine method. Eventually the proposed method was applied to vibration signals measured on an experimental bearing test rig. The results confirm that the method can be successfully applied to distinguish between healthy and faulty bearings even if the shaft speed changes dramatically.

\section{Keywords}

Rolling bearings fault detection, Teager-Kaiser energy operator (TKEO), one-class support vector machine, performance improved ensemble empirical mode decomposition (PIEEMD), cointegration, time-varying operating conditions

\section{Introduction}

Many research efforts have been focused on fault diagnosis and detection of rolling bearings, since they constitute one the most important elements of rotating machinery. Most of the diagnostic methods that have been developed up to date can be applied for the case of stationary working conditions only (constant speed and load). Randall and Antoni [1] treat broadly the background of some powerful diagnostic methods for roller bearings in a very useful tutorial paper. However, bearings often work at time-varying conditions such as wind 
turbine supporting bearing, mining excavator bearing, run-up and run-down processes. Damage identification for bearings working under non-stationary operating conditions, especially for early/small defects, requires the use of appropriate techniques, which are generally different from those used for the case of stationary conditions, in order to extract fault-sensitive features which are at the same time insensitive to operational condition variations. So far, some diagnostic techniques have been proposed for time-varying conditions. Among them, the order tracking method has been widely used for cases of speed variations. But there are still two main drawbacks of the order tracking method: limitation on the speed variation and the additional measurement costs introduced by providing the phase signal. The method can effectively detect faults when the speed variation is limited and an extra device, such as an encoder or a tachometer has to be used to provide a phase reference signal. Thus, many studies suggest the improvement of the order tracking method [2-7] and its development so that it can be applied tacho-less [8-9]. Li et al. [10] presented a method based on order tracking and Teager-Huang Transform (THT) for detection of bearing faults in gearbox under non-stationary run-up of gear drives. Then, Li [11] combined computed order tracking technique with bi-spectrum analysis. In both methods a speed transducer was used to measure rotational speed. Urbanek et al. [12] introduced the method called averaged instantaneous power spectrum as a time-frequency representation of selected cyclic components and tested on a selected case of wind turbine drive train fault. However, they believed that averaging can be applied when dealing with limited fluctuations of operational conditions. Cocconcelli et al. [13] applied another method for damage detection of roller bearing in direct-drive motors. They used the marginal time integration of the averaged Short Ttime Fourier transform (STFT) spectrogram as a simple indicator of damage. As the AC motor was controlled by a drive, they were able to use the speed profile in their methodology. They also applied the spectral kurtosis and the energy distribution for damage identification of a brushless AC motor using the angular velocity [14]. 
However, for the discussed methods, recording the speed requires additional instrumentation, which increases the measurement and the computational costs and adjustment problems.

In another approach, Zimroz et al. [15] used a two dimensional space made of the peak-to-peak amplitude of vibration signal and the generated power for diagnosis of the main bearing of a wind turbine working under non-stationary conditions. However, in most applications, there is no additional data that can be measured during the process (as e.g. the generated power) apart from the measured acceleration signals. And it should be kept in mind that for the case of wind turbines, the speed variation of the bearings is limited and called fluctuation (less than $30 \%$ [16]) in comparison with some time-varying conditions such as fast run up conditions.

Cointegration is a statistical concept which can be used for testing the existence of statistically significant relation between two or more non-stationary time series. It is looking for linear stationary combinations within non-stationary time series. Engle and Granger [17] formulated one of the first tests for cointegration. Johanson proposed a maximum likelihood approach for finding stationary linear combinations of non-stationary variables, which have the same order of integration [18]. Applications of cointegration to finance may be found in [19-23]. Cointegration has been recently applied for structural health monitoring (SHM) purposes to remove the non-stationarity produced by environmental variations such as temperature, wind and humidity [24-27]. Cross and Worden discussed the application of cointegration to engineering data [25]. Cross et al. [26] successfully applied cointegration for SHM purposes, where it was used to detect the introduction of damage in a composite plate. Antoniadou et al. applied the Hilbert-Huang and Teager- Kaiser transforms to extract relevant information from the acquired signals and subsequently used cointegration to remove the nonstationarity produced by variation of the environmental conditions [24]. Worden et al. demonstrated how a 
multi-resolution approach to cointegration can enhance the damage assessment capability for the case of a composite plate [27].

The Teager-Kaiser energy based feature extraction method proposed by Tabrizi et al [39] is able to successfully identify the early damage level of roller bearings operating in stationary conditions. In this study a new approach is proposed to detect the state of rolling bearings operating in conditions of time-varying speed. Contrary to the afore-mentioned methods, we collected only acceleration signals and it does not require any additional measurements and/or instrumentation. Furthermore, it works even for cases when the speed changes rapidly (for example in a run-up start). The methodology is introduced in two parts, signal analysis and pattern recognition. The signal acquired is divided into segments and each segment is broken down into some elementary modes the Intrinsic Mode Functions (IMFs) using the Performance Improved Ensemble Empirical Mode Decomposition (PIEEMD) proposed by some of the authors of this study for stationary operating condition [28]. Then, three dimensional feature vectors are created by applying the Teager-Kaiser Energy Operator (TKEO) to the cointegrated residuals of the first three IMFs. The feature vectors obtained from healthy bearing signals are further utilized as input to construct a separating hyperplane for a one-class Support Vector Machine (SVM). The SVM can be trained to categorize signals coming from healthy and faulty bearings. It is shown that the proposed method can successfully identify signals from healthy and faulty bearings.

\section{The rest of the paper is organized as follows:}

Signal analysis and pattern recognition (one-class SVM) methods are introduced in section 2.1 and 2.2 , respectively. The whole process for roller element bearing fault detection is explained in 2.3. The experimental setup and the data-acquisition process are presented in section 3. The application of the new approach to the acquired data together with some results is discussed in section 4. Eventually, the paper concludes with a discussion in section 5 . 


\section{1 .Signal analysis}

In order to extract signal features each collected signal is divided into segments. Then, several methods are applied. The signal analysis techniques are detailed below.

\subsubsection{Segmentation}

The first step of the method proposed is to divide the whole signal into $n$ segments with equal length as follows:

$$
\mathrm{x}(t)=\sum_{z=1}^{n} x_{z}(t)
$$

where $z=1, \ldots, n$ is the number of segments. Each segment should include many data points to carry the fault detection information required. On the other hand, the number of segments should be high to extract stationarity (as it will be discussed in section 2.1.3). In this study each collected signal is divided into 5 segments $(n=5)$ so that each segment includes 6000 data points and is demonstrated that is appropriate for fault detection purpose.

\subsubsection{The application of the Ensemble empirical mode decomposition (EEMD)}

In this section each segment is decomposed into IMFs. The empirical mode decomposition (EMD) is an adaptive, data driven technique to decompose a signal into several IMFs [29]. In order to solve the mode mixing problem of the EMD, which is defined as any IMF consisting of oscillations of dramatically disparate scales, often caused by intermittency of the driving mechanisms, the Ensemble empirical mode decomposition 
(EEMD) is a technique recently proposed [30]. The EEMD decomposition steps of each segment can be summarized as follows

a) First random white noise signals are added to each segment $x_{z}(t)$ as shown below

$$
x n_{z j}(t)=x_{z}(t)+A m p \cdot n_{z j}(t) \quad j=1,2, \ldots, M
$$

where $A m p$ is the amplitude of added white noise and $M$ is a pre-determined number of trials and different random white noise is added each time.

b) The obtained signals $\left(x n_{z j}(t)\right)$ are decomposed into IMFs using the EMD as detailed below:

$$
x n_{z j}(t)=\sum_{i=1}^{N_{j}} c_{i z j}(t)+r_{N_{j}}(t)
$$

where $c_{i z j}$ represents the i-th IMF of the j-th trial, $r_{N_{j}}$ denotes the residue of $\mathrm{j}$-th trial and $N_{j}$ is the number of IMFs within the $\mathrm{j}$-th trial.

c) The ensemble means of the obtained IMFs , $c_{i j z}$, are calculated: 


$$
I M F_{i z}(t)=\left(\sum_{j=1}^{M} c_{i j z}\right) / M
$$

where $i=1,2, \ldots, L, M$ is the minimum number of IMFs among all the trials and $z$ is segment number. Thus, for example, $I M F_{11}, \ldots, I M F_{L 1}$ are the $L$ IMFs obtained by decomposing the first segment and $I M F_{12}, \ldots, I M F_{L 2}$ are the $L$ IMFs obtained by decomposing the second segment and so on.

Adding noise aims to affect the extrema of the original signal so that the intermittency of the components will be removed. In order to improve the performance of the EEMD, an adaptive method called Performance Improved EEMD (PIEEMD) was proposed by Tabrizi et al. [28] to determine the appropriate amplitude of added noise. After adding a random white noise, by applying the signal to noise ratio (SNR) definition (see equation (5)), the amplitude value for each data point of a segment is obtained using equation (6). The SNR is considered as a constant predetermined value $(\mathrm{SNR}=10)$ in equation $(6)$.

$S N R=20 \log ($ Signal amplitude/Noise amplitude)

$$
A m p_{z j}(t)=10^{-\left(\frac{S N R}{20}\right)}\left(x_{z}(t) / n_{z j}(t)\right)
$$

where $\mathrm{z}$ corresponds to the segment $, z=1,2 \ldots n$ and $j=1,2 . ., M$ is the number of trials. 


\subsubsection{Cointegration}

The cointegration technique is used to find linear stationary combination of the corresponding IMFs (obtained in section 2.1.2.). Two or more non-stationary time series (with the same integrated order) are said to be cointegrated if a linear combination of them is stationary. A signal/stochastic process $\boldsymbol{y}_{\boldsymbol{t}}$ is said to be integrated of order $\mathrm{d}\left(\boldsymbol{y}_{\boldsymbol{t}} \sim \boldsymbol{I}(\boldsymbol{d})\right)$, if it becomes stationary after $d$ times differencing. Thus, a time series $\boldsymbol{y}_{\boldsymbol{t}}$ will be integrated of order 1, if its first difference is stationary $\left(\Delta \boldsymbol{y}_{\boldsymbol{t}} \sim \boldsymbol{I}(\mathbf{0})\right)$. The order of integration can be estimated by a stationarity test using the Augmented Dickey-Fuller (ADF) test [31].

Now we will form new signals $Y_{i t}$ from the obtained IMFs. A signal $Y_{i t}$ is made of $n$ non-stationary IMFs $\left(I M F_{i 1}, \ldots, I M F_{i n}\right)$ obtained from the $n$ signal segments xn (see equation (4)).

$$
Y_{i t}=\left(\begin{array}{c}
I M F_{i 1} \\
\vdots \\
I M F_{i n}
\end{array}\right)
$$

where $i=1, \ldots, m$ is the order of IMFs and $m$ is the number of IMFs selected. For example, when $i=1$, it means that $Y_{1 t}$ is made of the first IMFs of all $\mathrm{n}$ segments $\left(I M F_{11}, \ldots, I M F_{1 n}\right)$ and when $i=1, Y_{2 t}$ is made of second IMFs of all segments $\left(I M F_{21}, \ldots, I M F_{2 n}\right)$.

The series $Y_{i t}$ is said to be cointegrated with $r$ cointegrating vectors $(0<r<n)$ if there exists a $(n \times r)$ matrix $\beta$ such that the cointegrating residual $u$ is 0 integrated. 


$$
\beta^{\prime} Y_{i t}=\left(\begin{array}{c}
\beta_{1}^{\prime} Y_{i t} \\
\vdots \\
\beta_{r}^{\prime} Y_{i t}
\end{array}\right)=\left(\begin{array}{c}
u_{i 1} \\
\vdots \\
u_{i r}
\end{array}\right)
$$

Where $\beta^{\prime}$ is introduced as transpose of $\beta$.

The cointegration test based on Johansen procedure [18], tests for the existence of $r, 0 \leq \mathrm{r}<\mathrm{n}$, cointegrating vectors $\left(\beta_{1}, \ldots, \beta_{r}\right)$. It determines which of the cointegrating vectors creates the most stationary linear combination.

In order to find the cointegrating vectors, first we establish the residuals of the following regressions:

$$
\begin{aligned}
& \Delta Y_{i t}=\sum_{t^{\prime}=1}^{p} \Phi_{1 t^{\prime}} \Delta Y_{i\left(t-t^{\prime}\right)}+\widehat{U}_{i t} \\
& Y_{i(t-1)}=\sum_{t^{\prime}=1}^{p} \Phi_{2 t^{\prime}} \Delta Y_{i\left(t-t^{\prime}\right)}+\widehat{V}_{i t}
\end{aligned}
$$

where $p$ denotes the model order, $\widehat{U}_{t}$ and $\widehat{V}_{t}$ denote the residuals and $\Phi_{1}$ and $\Phi_{2}$ are multiplication matrices. Then, the cointegrating vectors are determined as eigenvectors of the following eigenvalue problem:

$$
\left|\lambda_{i} S_{i 11}-S_{i 10} S_{i 00}^{-1} S_{i 01}\right|=0
$$


where $S_{i 00}=\frac{1}{T} \sum_{t=1}^{T} \widehat{U}_{i t} \widehat{U}_{i t}^{\prime}, S_{i 01}=\frac{1}{T} \sum_{t=1}^{T} \widehat{U}_{i t} \widehat{V}_{i t}^{\prime}, S_{i 10}=\frac{1}{T} \sum_{t=1}^{T} \widehat{V}_{i t} \widehat{U}_{i t}^{\prime}, S_{i 11}=\frac{1}{T} \sum_{t=1}^{T} \widehat{V}_{i t} \widehat{V}_{i t}^{\prime}$ are the sample covariance matrices.

The eigenvector corresponding to the largest eigenvalue is the most stationary cointegration vector. The test statistic (see equation (12)) is applied to test the hypothesis.

$$
\operatorname{Tstat}_{i}\left(r_{0}\right)=-T \sum_{q=r_{0}+1}^{R} \ln \left(1-\hat{\lambda}_{i q}\right)
$$

where $i=1, \ldots, m$ is the order of IMFs and $m$ is the number of IMFs selected, $\hat{\lambda}_{i q}$ denotes the estimated eigenvalues and $R$ is the number of eigenvalues obtained from equation (11).

P-value is defined as the right-tail probabilities of the test statistics. Thus, it is the probability under the assumption of the null hypothesis. The null hypothesis is rejected if the P-value is less that a pre-determined significance level, which is usually set to $5 \%(0.05)$. The critical values, tabulated by Johansen [18], were calculated based on 5\% P-value. In this case, the null hypothesis is rejected, when the trace statistic value become less than critical value.

First the null hypothesis $\left(\mathrm{H}_{0}\left(r_{0}=0\right)\right)$ is tested against the alternative hypothesis $\left(\mathrm{H}_{1}\left(r_{0}>0\right)\right)$. If the null hypothesis is accepted then there are no cointegrating vectors. If the null hypothesis is rejected then there is at least one cointegration vector and the test is continued to test $\mathrm{H}_{0}\left(r_{0}=1\right)$ against $\mathrm{H}_{1}\left(r_{0}>1\right)$. There exists only one cointegrating vector, if the null hypothesis is accepted. Otherwise, it is concluded that there are at least two cointegrating vectors. The procedure is continued until the null hypothesis is accepted. 


\subsubsection{Teager-Kaiser Energy Operator (TKEO)}

The energy of a signal is the sum of squared absolute value of the signal over a time, which is not the instantaneous summed energy. Kaiser observed that a second order differential equation is the energy required to generate a simple sinusoidal signal varies with both amplitude and frequency [32]. In order to estimate the instantaneous energy of a signal $\mathrm{x}(\mathrm{t})$, Teager-Kaiser Energy Operator (TKEO) is used as an energy tracking operator as follows (Maragos, 1993):

$\Psi[x(t)]=A^{2}=\dot{x}^{2}(t)-x(t) \ddot{x}(t)$

where $\dot{x}(t)$ and $\ddot{x}(t)$ are the first and the second time derivatives of $\mathrm{x}(\mathrm{t})$, respectively.

For a discrete signal, using differences to approximate differentiation, the TKEO can be developed as [33]:

$$
\psi\left[u_{i j}(t)\right]=u_{i j}^{2}(t)-u_{i j}(t+1) u_{i j}(t-1)
$$

where $t$ is the discrete time index.

As at any instant, only three consecutive values are needed to estimate the instantaneous TKEO, it is adaptive to the instantaneous changes in signals and is able to resolve transient events.

In the method proposed the TKEO is applied to the $r$ stationary cointegrated residuals $\left(u_{i 1} \quad \ldots \quad u_{i r}\right)$ obtained in section 2.1.3. The sum would be a value and is obtained as follows:

$$
T K E_{i j}=\sum \psi\left(u_{i j}\right)
$$

where $i=1, \ldots, m$ is the number of IMFs selected and $j=1, \ldots, r$ is the number of cointegrated residuals. 


\subsubsection{Creating feature vectors}

Feature vectors used as input for the consequent pattern recognition method are formed based on the values calculated in equation (15). In this study three IMFs are selected, in order to have enough information and on the other hand less dimensional feature vectors [34].

$$
F_{j}=\left[\begin{array}{lll}
T K E_{1 j} & T K E_{2 j} & T K E_{3 j}
\end{array}\right]
$$

where $j=1, \ldots, r$ is the number of cointegrated residuals. For example, $F_{1}$ is the three dimensional feature vector created using the first residual and $F_{2}$ is the feature vector created using the second residual. Thus, for each signal we find $r$ feature vectors.

Then, the feature vectors obtained are normalized by dividing them to their Euclidean norm as follows:

$$
F V n_{j}=\left[T K E_{1 j} / \operatorname{norm}\left(T K E_{1 j}\right) \quad T K E_{2 j} / \operatorname{norm}\left(T K E_{2 j}\right) \quad T K E_{3 j} / \operatorname{norm}\left(T K E_{3 j}\right)\right]
$$

Which can be represented as follows so that each dimension would be a value:

$F V n_{j}=\left[\begin{array}{lll}F V_{1 j} & F V_{2 j} & F V_{3 j}\end{array}\right]$ 


\subsection{Pattern recognition}

In this part a pattern recognition method is applied in order to recognize between healthy and faulty bearing or rather the signals that come from them. For the purpose the one class support vector machine method is applied. The method is briefly described below.

\subsubsection{One-class support vector machine}

The support vector machine (SVM) introduced by Vapnik is a relatively new computational learning statistical PR method based on statistical learning theory [35]. It is applied for recognizing between two classeshealthy and faulty signals. The method requires a relatively small training samples for the learning phase. Thus, the method accommodates the fact that acquiring a sufficient number of faulty signals is not applicable in practice, and it has been successfully used in a number of fault diagnosis problems. The one-class SVM

Insert [Figure 2- Classification by one-class SVM (from [36])] somewhere here

proposed by Scholkopf et al. is a powerful technique which constructs a separating hyperplane between two classes of patterns using data from one of the classes only. It classifies the patterns from the other class as outliers [37]. It constructs a hyperplane around the data, such that its distance to the origin (called Margin) is maximal among all possible hyperplanes and can be adopted for anomality detection, as shown in Figure 2.

For each residual, the feature vectors $F V n_{j}$ obtained from the healthy bearing signals (using equation (18)) are used to construct the hyperplane. As there are a number of features for each residual, we use $l F V n_{j k}$ vectors as features, where $j$ denotes residual number and $k=1, \ldots, l$ is the feature number used for training. 
The SVM can be also applied in case of non-linear classification by mapping the data onto a high dimensional feature space $\left(\phi\left(F V n_{j k}\right)\right)$, where the linear classification is then possible. In real problems, an exact line dividing the data is usually difficult to obtain, so, by introducing the slack variable $\left(\xi_{k}\right)$ in equation (19) and ignoring few outlier data points a smooth boundary can be created.

The Margin is defined as [37]:

$$
\text { Margin }=\rho /\|w\|
$$

where $w$ and $\rho$ are the weight vector and the offset parameterizing the hyperplane.

In order to separate the data set from the origin, the following quadratic optimization problem must be solved [37]:

$$
\operatorname{minimize}\left(\frac{1}{2}\|w\|^{2}+\frac{1}{v l} \sum_{k=1}^{l} \xi_{k}-\rho\right)
$$

$$
\text { subject to }\left\{\begin{array}{c}
y_{k}\left(w \cdot \phi\left(F V n_{j K}\right)\right) \geq \rho-\xi_{k} \\
\xi_{k} \geq 0
\end{array}\right.
$$

where $j=1, \ldots, r$ is the number of residuals, $k=1, \ldots, l$ is the features used to train the model, $y_{k}$ is a vector includes the label of each feature $(+1$ for the features of healthy bearing signals and -1 for the features of faulty bearing signals), $\xi_{k}$ is the slack variable and measuring the distance between the hyperplane and the examples 
that laying in the wrong side of the hyperplane (Figure 3), $v$ is the regularization parameter and represents an upper bound on the fraction of outliers (training errors) and a lower bound on the fraction of support vectors (the nearest features to the hyperplane) with respect to the number of training samples. It is a variable taking values between 0 and 1 that monitors the effect of outliers (hardness and softness of the boundary around data). By applying a kernel function as the inner product of mapping functions $\left(K\left(F V n_{j k}, F V n_{j k},\right)=\left(\phi\left(F V n_{j k}\right)\right.\right.$. $\left.\phi\left(F V n_{j k^{\prime}}\right)\right)$, it is not necessary to explicitly evaluate mapping in the feature space, where $, k^{\prime}=1, \ldots, l$. Various kernel functions can be used, such as linear, polynomial or Gaussian RBF (Radial basis function). In this study, RBF kernel function, a common function in fault detection problems, is applied:

$K\left(F V n_{j k}, F V n_{j k^{\prime}}\right)=\exp \left(-\gamma\left\|F V n_{j k}-F V n_{j k},\right\|^{2}\right)$

Insert [Figure 3- The slack variables $\xi_{i}$ and support vectors (data points with circles around them) (from

\section{[38]).] somewhere here}

Introducing Lagrange multipliers the dual problem is formulated as:

$$
\operatorname{minimize} \frac{1}{2} \sum_{k, k^{\prime}=1}^{l} \alpha_{k} \alpha_{k^{\prime}} K\left(F V n_{j k}, F V n_{j k^{\prime}}\right)
$$

subject to $\left\{\begin{array}{l}0 \leq \alpha_{k} \leq \frac{1}{v l} \\ \sum_{k=1}^{l} \alpha_{k}=1\end{array}\right.$ 
If $v$ approaches 0 , the upper boundaries on the Lagrange multipliers tend to infinity, so the second inequality constraint in (equation (22)) becomes void. Furthermore, the penalty for errors becomes infinite, so the algorithm returns to the corresponding hard margin algorithm.

The feature vectors $(\mathrm{FV})$ which achieve positive, non-zero multipliers called support vectors $S V_{j i}$ where $i$ corresponds the number of support vectors (SV). Then, offset is calculated as follows:

$$
\rho=\left(w \cdot \phi\left(S V_{j i}\right)\right)=\sum_{k=1}^{l} \alpha_{k} K\left(F V n_{j k}, S V_{j i}\right)
$$

Accordingly the non-linear decision function for labelling new samples $F V n_{j}$ is represented as follows:

$$
f\left(F V n_{j}\right)=\operatorname{sign}\left(\sum_{k=1}^{l} \alpha_{k} K\left(F V n_{j k}, F V n_{j}\right)-\rho\right)
$$

The classification parameters ( $\gamma$ and $v$ ), which must be determined, are optimized by using cross validation method.

\subsection{The whole methodology}

Eventually the method suggested can be summarized as shown in Figure 3.

Insert [Figure 3- The cointegration based methodology suggested in this study for roller bearing fault 


\section{Method validation and verification}

For the purpose of demonstration and validation of the suggested methodology, experimental data (only acceleration signal) was obtained from the test rig assembled at the DASM Group in the Department of Mechanical and Aerospace Engineering of Strathclyde University which is shown in Figures 4(a) and (b). As discussed in section 2.3 we do not require any data except for the acceleration signals. The test rig consists of a shunt DC motor (1 hp and $2000 \mathrm{rpm}$ ), bearing assembly and a mechanical loading system. In order to acquire acceleration signals, the MONITRAN accelerometer (MTN/1120 model) was fixed to the bearing supports by magnetic coupling. The bearings used in the experiment were SKF deep grooves 6308. Two bearing conditions were considered in this study healthy and inner race faulty. The fault was considered a small notch on the inner ring created using spark erosion to simulate a flaking or spalling fault. The width was considered less than $1 \mathrm{~mm}$ and the depth was almost 100 microns. 20 signals were acquired for each bearing condition. Signals were sampled at $1.3 \mathrm{kHz}$ and each had a 25 seconds time length. For all the acceleration signals acquired, the run up time varying operating condition was considered by varying the speed manually from 150 to $1500 \mathrm{rpm}$. Thus, speed variation considered is significant with different speed rates to demonstrate that the results are independent to speed rate. The faults considered here were very small notches. Thus, the primary interest of this study is to detect very small faults at a rather early stage. Two acquired signals during run-up condition from the healthy and damaged bearings are displayed in Figure 5.

The process of the method follows the steps detailed above in section 2.3. 
Insert [Figure 4. a,b) The test rig used to collect the signals coming from healthy and faulty bearings.]

\section{somewhere here}

Insert [Figure 5. Two acquired signals during run-up condition a) from the healthy bearing b) from the damaged bearing.] somewhere here

\section{Results and discussion}

In this section we present and discuss some results from the application of the suggested method. Firstly the results of the application of the Teager-Kaiser energy (TKE) based feature extraction method proposed by Tabrizi et al. [39] are provided in order to test the identifiability of the measured signals. Subsequently the method suggested in this study (cointegration based method) will be applied to the same signals in order to see the effect of the cointegration residuals on the resulting classification. In the TKE based method [39], a signal is decomposed into IMFs using the performance improved EEMD (PIEEMD) and then the TKE operator is applied to the first three IMFs to obtain three dimensional feature vectors. After normalizing, one obtains three feature vectors called $\boldsymbol{T K E n _ { 1 }}, \boldsymbol{T K E n _ { 2 }}$ and $\boldsymbol{T K E n _ { 3 }}$. The results of applying TKE based method to the signals collected in time-varying operating condition (run-up) are shown in Figure 6. The Figure presents all the three features on a 3d plot (Figure 6(a)) and different combinations of the features $\boldsymbol{T K E n _ { 1 }}, \boldsymbol{T K E n _ { 2 }}$ and $\boldsymbol{T K E n}_{\mathbf{3}}$ (Figure 6 (b), (c) and (d)). Although this method is known to be a powerful tool for detection of small faults, it can be seen that for the case of non-stationary operational conditions the feature vector points are not well separated and it is impossible to determine the condition of the bearing. Thus in this case for a variable motor speed the above method fails to provide reliable fault detection.

Insert [Figure 6. The normalized TKE using the combined method consists of PIEEMD (as the decomposition technique) and TKEO (to create feature vectors).] somewhere here 
Secondly, it is investigated if and to what extent the cointegration based method proposed in this study (section 2.3) is able to recognize the state of the bearings. As detailed in the section 2.3, first, each signal is divided into five segments so that each segment includes 6000 data points. Then, each segment is decomposed by the PIEEMD algorithm. The results for healthy and faulty signals are given in Figures 7 and 8 . The cointegration procedure is then carried out for all the five IMFs corresponding to each segment to obtain one or more cointegrating vectors.

The results of Johansen test for cointegration for the healthy bearing signal (displayed in Figure 5(a)) are given in Table 1. From Table 1, the results regarding the existence of cointegration relations among a set of IMFs are given. The table shows that there are four linear combinations of the IMFs labeled under the null ranks column (i.e r0, r1 ...r3). The other columns give information whether each of these linear combinations indicates cointegration among the IMFs or not. The null hypothesis of the test suggests that there is no cointegration among the IMFs. In this table the $h$ values given in column 2 of Table 1 are all equal to 1 , which rejects the null hypothesis. Consequently, the problem is full ranked since all the null hypothesis are rejected. The right-tail probabilities Prt (i.e P-values given in column 5) are considerably lower than the significance level (which is 0.05). This indicates that there is enough evidence of the stationarity of the cointegrating vector (since there is a cointegration relation among the IMFs).

Insert [Figure 7. The IMFs of the first segment of the healthy signal (Figure 5(a)) obtained performance improved EEMD (PIEEMD).] somewhere here

Insert [Figure 8. The IMFs of the first segment of the faulty signal (Figure 5(b)) obtained using performance imperoved EEMD (PIEEMD).] somewhere here 
Insert [Table 1. The results of cointegration for the first IMFs of five segments of the healthy signal shown in Figure 5(a)] somewhere here

Insert [Figure 9. The cointegrating vectors obtaind from first IMFs $\left(\begin{array}{lll}I M F_{11} & \ldots & I M F_{15}\end{array}\right)$ of the five segments of the signal shown in Figure 5(a).] somewhere here

Insert [Figure 10. The TKEO applied to the cointegrated residuals obtained from $\left(\begin{array}{lll}I M F_{11} & \ldots & I M F_{15}\end{array}\right)$, $\left(\begin{array}{lllll}I M F_{21} & \ldots & I M F_{25}\end{array}\right)$ and $\left(\begin{array}{lll}I M F_{31} & \ldots & I M F_{35}\end{array}\right)$, respectively.] somewhere here

The values of the other parameters in Table 1 (test statistics values, critical values and Eigenvalues) give information about the stationarity for each cointegration residual. The Eigenvalues are arranged in ascending order corresponding to the order of null ranks ( $\mathrm{r} 0, \mathrm{r} 1, \mathrm{r} 2$ and $\mathrm{r} 3$ ). The four stationary cointegration residuals are shown in Figure 9.

Eventually, the TKEO is applied to each cointegration residual (equation (14)), and the results are shown in Figure 10. The three dimensional feature vectors are created according to equation (15). After normalizing (see equation (16)), the feature vectors used in the classification process are obtained (equation (17)). Thus, for each signal, we are in possession of one three dimensional feature vector.

The visualization of the 3-dimensional feature vectors and their projections corresponding to the healthy and faulty bearings for each cointegration residual are shown in Figures 11 to 14. A very good separation between the healthy and the faulty classes obtained from the first and the second cointegrated residuals can be appreciated. For each cointegration relation, there are 20 feature vectors developed for each class. From the class of healthy bearings, $80 \%$ of the feature vectors (i.e 16 out of 20) are used as training input for the oneclass SVM method (see section 2.2.1). Consequently, the constructed hyper-plane is utilized for determining 
Insert [Figure 11. The three dimensional feature vectors obtained from first cointegrated residual.] somewhere here

Insert [Figure 12. The three dimensional feature vectors obtained from the second cointegrated residual.] somewhere here

Insert [Figure 13. The three dimensional feature vectors obtained from the third cointegrated residual.] somewhere here

Insert [Figure 14. The three dimensional feature vectors obtained from the fourth cointegrated residual.] somewhere here

the condition of the new test vectors. The test sample contains the remaining $20 \%$ of the healthy and all faulty feature vectors. Since, the signals were collected in different speed variations, the healthy signals acquired are different, which means that the training samples ( $80 \%$ of the healthy features) and healthy test samples (remaining $20 \%$ of healthy features) are not similar.

The classification rate is shown in the Table 2. It can be seen that for all the cases (all cointegration residuals), the success rates for the training and the test samples are $100 \%$ when the first and the second cointegration residuals are used, as this result was expected based on Figures 11 and 12. However, when the third or the fourth cointegration residuals are used the features vectors from the test sample are not well separated, but the success rate for the training sample is still $100 \%$. When using the third cointegration residual, there are two false-positive cases (i.e two healthy samples that are miss-classified as faulty). When the fourth cointegration residual is used four out of the 24 samples were miss-labeled (one false -positive case and three false-negative ones (i.e three faulty FV are misclassified as healthy). False-positive yields to implement a maintenance 
procedure whereas consequence of false-negative is to run the machine when it is faulty and has to be stopped to be repaired.

Insert [Table 2. The classification results for different cointegrated residuals] somewhere here

The margin obtained for each classification is also indicated in Table 2. It can be seen that using the feature vectors obtained from the first or the second residuals vector provides a more reliable hyperplane, as they have higher margin values, while the margin values for the third and fourth residuals fall down and accordingly the classification rate deteriorates.

Since the method proposed is anomaly detection (one-class SVM), all data acquired from faulty bearing was used to test the method. In order to demonstrate the ability of the method, new signals ( 20 signals) were collected from a new healthy bearing and based on the results obtained the method recognized perfectly the state new healthy bearing.

On the basis of the results discussed above it can be appreciated that the implementation of the method proposed in this study, a very good/ perfect recognition of the bearing state can be achieved, even when the shaft speed changes significantly.

\section{Conclusions}

This study proposes a roller element fault detection method, which is based on the statistical concept of cointegration. The cointegration is used for the purposes of detecting the stationary content of the signal by finding the linear stationary combinations of its elements. The signal is first divided into segments and each segment is broken down into elementary modes, the IMFs, using the Performance Improved Ensemble 
Empirical Mode Decomposition (PIEEMD). Subsequently, three dimensional feature vectors are created by applying the Teager-Kaiser Energy Operator (TKEO) to the cointegrated residuals of the first three IMFs.

The suggested method can work for constant operational speed but unlike most other fault detection methods for roller element bearings it can distinguish between healthy and faulty bearings even when the speed is significantly changing. Most roller element fault detection methods that can operate in speed varying conditions require additional instrumentation like e.g a tachometer or encoder. Some of these methods, like the order tracking one, can detect the damage when the speed variation is limited. The method suggested in this study does not put any constraints on the speed variation and can work even when the speed changes rapidly (for example in a run-up start). This is a very important merit of the proposed method since most rotating machineries operate with time varying speed and the application of this method provides a procedure for online fault monitoring, without putting the machinery into parts.

This study validates the suggested method by applying it on the data obtained from an experimental setup operating in run-up condition. The suggested method uses a training pattern recognition procedure in order to recognize between healthy and faulty bearing signals. $80 \%$ of the acquired signals are used for training purposes and the rest are used as a testing sample.

The results given in the previous paragraph show a $100 \%$ correct classification for the signals from the testing sample and $100 \%$ correct classification for the testing sample as well when the most stationary cointegration residuals (the first and the second one) are used to obtain the feature vectors. When the residuals with lower/weaker stationarity are used, the results deteriorate slightly but still the majority of the signals are correctly recognized.

The suggested methodology can be further developed for the purposes of qualification (fault type recognition) and eventually quantification purposes using the multi class SVM. But even in its proposed state 
as a detection method this is the first methodology of such kind which can operate under time varying speed (run-up) without requiring additional instrumentation.

\section{Acknowledgement}

This work has been partially carried out in the framework of the GREAT2020 - phase II, project.

\section{References}

[1] Randall RB, Antoni J (2011) Rolling element bearing diagnostics - A tutorial. Mechanical Systems and Signal Processing 25:485-520.

[2] Andre H, Daher Z, Antoni J, Rémond D (2010) Comparison between angular sampling and angular resampling methods applied on the vibration monitoring of a gear meshing in non-stationary conditions. In: 24th International Conference on Noise and Vibration engineering (ISMA2010), Leuven, Belgium, 2022 September 2010, pp.2727-2736.

[3] Borghesani P, Ricci R, Chatterton S, Pennacchi P (2013) A new procedure for using envelope analysis for rolling element bearing dignostics in variable operating conditions. Mechanical systems and signal processing 38:23-35.

[4] Borghesani P, Pennacchi P, Chatterton S, Ricci R (2014) The velocity synchronous discrete Fourier transform for order tracking in the field of rotating machinery. Mechanical systems and signal processing 44:118-133.

[5] Coats MD, Randall RB (2014) Single and multi-stage phase demodulation based order-tracking. Mechanical Systems and Signal Processing 44:86-117.

[6] Renaudin L, Bonnardot F, Musy O, Doray JB, Remond D (2010) Natural roller bearing fault detection by 
angular measurement of true instantaneous angular speed. Mechanical Systems and Signal Processing 24: 1998-2011.

[7] Villa LF, Reñones A, Perán JR, Miguel LJ (2011) Angular resampling for vibration analysis in wind turbines under non-linear speed fluctuation. Mechanical Systems and Signal Processing 25:2157-2168.

[8] Zhao M, Lin J, Xu X, Lei Y (2013) Tacholess envelope order analysis and its application to fault detection of rolling element bearings with varying speeds. Sensors 13:10856-10875.

[9] Coats MD, Randall RB (2012) Compensating for speed variation by order tracking with and without a tacho signal. In: 10th international Conference on Vibrations in Rotating Machinery (VIRM10), London, UK, 11-13 September 2012.

[10] Li H, Zhang Y, Zheng H (2010) Bearing fault detection and diagnosis based on order tracking and TeagerHuang transform. Mechanical science and technology 24 (3):811-822.

[11] Li H (2011) Order bi-spectrum for bearing fault monitoring and diagnosis under run-up condition. Journal of computers 6 (9):1994-2000.

[12] Urbanek J, Barszcz T, Zimroz R, Antoni J (2012) Application of averaged instantaneous power spectrum for diagnostics of machinery operating under non-stationary operational conditions. Measurement 45:1782-1791.

[13] Cocconcelli M, Zimroz R, Rubini R, Bartelmus W (2012a) STFT based approach for ball bearing fault detection in a varying speed motor. In: Fakhfakh T, Bartelmus W, Chaari F, Zimroz R, Haddar M (eds) Condition Monitoring of Machinery in Non-Stationary Operations. Springer Berlin Heidelberg, pp.41-50.

[14] Cocconcelli M, Zimroz R, Rubini R, Bartelmus W (2012b) Kurtosis over energy distribution approach for STFT enhancement in ball bearing diagnostics. In: Fakhfakh T, Bartelmus W, Chaari F, Zimroz R, Haddar M (eds) Condition Monitoring of Machinery in Non-Stationary Operations. Springer Berlin Heidelberg, 
pp.51-59.

[15] Zimroz R, Bartelmus W, Barszcz T, Urbanek J (2014) Diagnostics of bearings in presence of strong operating conditions non-stationarity—A procedure of load-dependent features processing with application to wind turbine bearings. Mechanical Systems and Signal Processing 46:16-27.

[16] Zimroz R, Urbanek J, Barszcz T, Bartelmus W, Millioz F, Martin N (2011) Measurement of instantaneous shaft speed by advanced vibration signal processing - application to wind turbine gearbox. Metrology and measurement systems XVIII:701-712.

[17] Engle R, Granger C (1987) Co-integration and error-correction: representation, estimation, and testing. Econometrica 55:251-276.

[18] Johansen S (1995) Likelihood-based inference in cointegrated vector autoregressive models. Oxford: Oxford university press.

[19] Alexander C (2001) Market Models: A Guide to Financial Data Analysis. John Wiley and Sons.

[20] Cochrane J (2001) Asset Pricing. New Jersey: Princeton University Press.

[21] MacKinnon J (1996) Numerical Distribution Functions for Unit Root and Cointegration Tests. Journal of Applied Econometrics 11:601-618.

[22] Mills T (1999) The Econometric Analysis of Financial Time Series. Cambridge: Cambridge University Press.

[23] Tsay R (2001) The Analysis of Financial Time Series. New York: John Wiley \& Sons.

[24] Antoniadou I, Cross EJ, Worden K (2013) Cointegration and the empirical mode decomposition for the analysis of the diagnostic data. Key engineering materials 569-570:884-891.

[25] Cross EJ, Worden K (2012) Cointegration and why it works for SHM, Journal of Physics: Conference Series 382:012046. 
[26] Cross EJ, Worden K, Chen Q (2011) Cointegration: a novel approach for the removal of environmental trends in structural health monitoring data. Proceedings of the Royal Society A: Mathematical, Physical and Engineering Science 467(2133):2712-2732.

[27] Worden K, Cross EJ, Antoniadou I, Kyprianou A (2014) A multi resolution approach to cointegration for enhanced SHM of structures under varying conditions- An exploratory study. Mechanical Systems and Signal Processing 47 (1-2):243-262.

[28] Tabrizi A, Garibaldi L, Fasana A, Marchesiello S (2015a) Early damage detection of roller bearings using wavelet packet decomposition, ensemble empirical mode decomposition and support vector machine. Meccanica 50 (3):865-874.

[29] Huang NE, Shen Z, Long SR, Wu ML, Shih HH, Zheng Q, Yen NC, Tung CC, Liu HH (1998) The empirical mode decomposition and the Hilbert spectrum for nonlinear and non-stationary time series analysis. Proceedings of the Royal Society of London Series A 454:903-995.

[30] Wu Z, Huang N (2009) Ensemble Empirical Mode Decomposition: A noise-assisted data analysis method. Advances in Adaptive Data Analysis 1(1):1-41.

[31] Dickey DA, Fuller WA (1979) Distribution of the estimators for autoregressive time series with a unit root. Journal of the American Statistical Association 74:427-431.

[32] Kaiser J (1990) On a simple algorithm to calculate the energy of a signal. In: The international conference on acoustics, speech and signal processing. Albuquerque, NM, 3-6 April 1990, pp. 381-384.

[33] Maragos P, Kaiser JF, Quatieri TF (1993) On amplitude and frequency demodulation using energy operators. IEEE Transaction Signal Processing 41:1532-1550.

[34] Tabrizi A, Garibaldi L, Fasana A, Marchesiello S (2015) Performance improvement of ensemble empirical mode decomposition for roller bearings damage detection. Shock and Vibration 2015 , Article ID 964805, 
10 pages.

[35] Vapnik AVN (1995) The nature of statistical learning theory. Berlin: Springer.

[36] Shin H J, Eom D-H, Kim S-S (2005) One-class support vector machines - an application in machine fault detection and classification. Computer \& Industrial Engineering 48:395-408.

[37] Scholkopf B, Williamson R, Smola A, Taylor JS, Platt J (2000) Support vector method for novelty detection. Advances in Neural Information Processing Systems 12:582-586.

[38] Bishop C.M. (2006) Pattern Recognition and Machine Learning (Information Science and Statistics). Springer.

[39] Tabrizi A, Garibaldi L, Fasana A, Marchesiello S (2015) A novel feature extraction for anomaly detection of roller bearings based on performance improved ensemble empirical mode decomposition and teager-kaiser energy operator. International Journal of Prognostics and Health Management 6 (Special Issue Uncertainty in PHM) 026: pages:10. 


\section{Figure captions}

Figure 1. Classification by one-class SVM (from [37]).

Figure 2. The slack variables $\xi_{i}$ and support vectors (data points with circles around them) (from [38]).

Figure 3. The cointegration based methodology suggested in this study for roller bearing fault detection working in operational variation condition.

Figure 4. a,b) The test rig used to collect the signals coming from healthy and faulty bearings.

Figure 5. Two acquired signals during run-up condition a) from the healthy bearing b) from the damaged bearing.

Figure 6. The normalized TKE using the combined method consists of PIEEMD (as the decomposition technique) and TKEO (to create feature vectors).

Figure 7. The IMFs of the first segment of the healthy signal (Figure 5(a)) obtained performance improved EEMD (PIEEMD).

Figure 8. The IMFs of the first segment of the faulty signal (Figure 5(b)) obtained using performance imperoved EEMD (PIEEMD).

Figure 9. The cointegrating vectors obtaind from first IMFs $\left(I M F_{11} \quad \ldots \quad I M F_{15}\right)$ of the five segments of the signal shown in Figure 5(a).

Figure 10. The TKEO applied to the cointegrated residuals obtained from $\left(\begin{array}{llll}I M F_{11} & \ldots & I M F_{15}\end{array}\right)$, $\left(\begin{array}{lllll}I M F_{21} & \ldots & \left.I M F_{25}\right)\end{array}\right)$ and $\left(I M F_{31} \quad \ldots \quad I M F_{35}\right)$, respectively.

Figure 11. The three dimensional feature vectors obtained from first cointegrated residual.

Figure 12. The three dimensional feature vectors obtained from the second cointegrated residual.

Figure 13. The three dimensional feature vectors obtained from the third cointegrated residual. 
Figure 14. The three dimensional feature vectors obtained from the fourth cointegrated residual.

\section{Table captions}

Table 1. The results of cointegration for the first IMFs of five segments of the healthy signal shown in Figure $5(a)$

Table 2. The classification results for different cointegrated residuals 


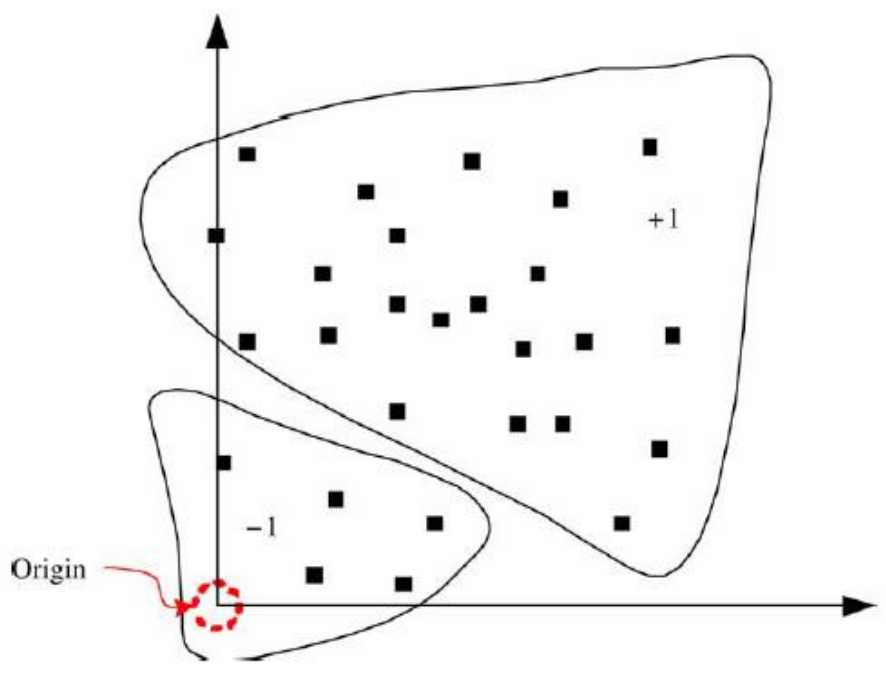

Figure 1. Classification by one-class SVM (from [37]). 


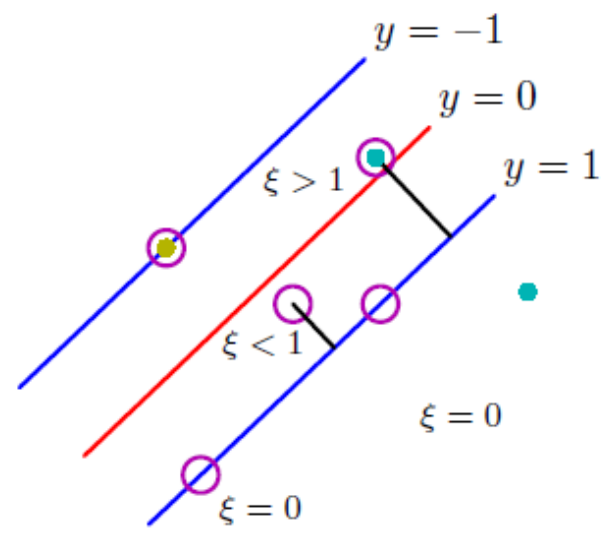

Figure 2. The slack variables $\xi_{i}$ and support vectors (data points with circles around them) (from [38]). 


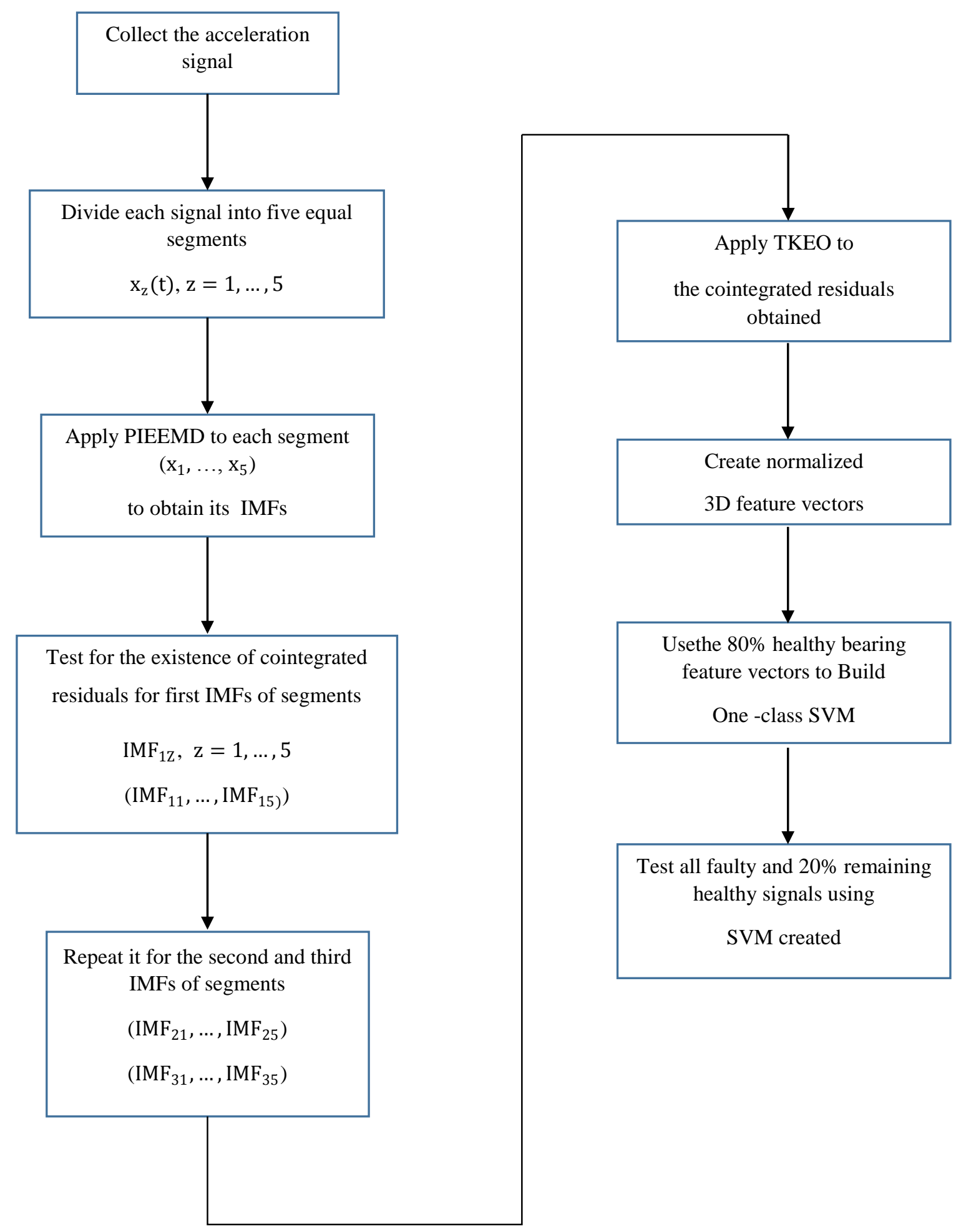

Figure 3. The cointegration based methodology suggested in this study for roller bearing fault detection working in operational variation condition. 


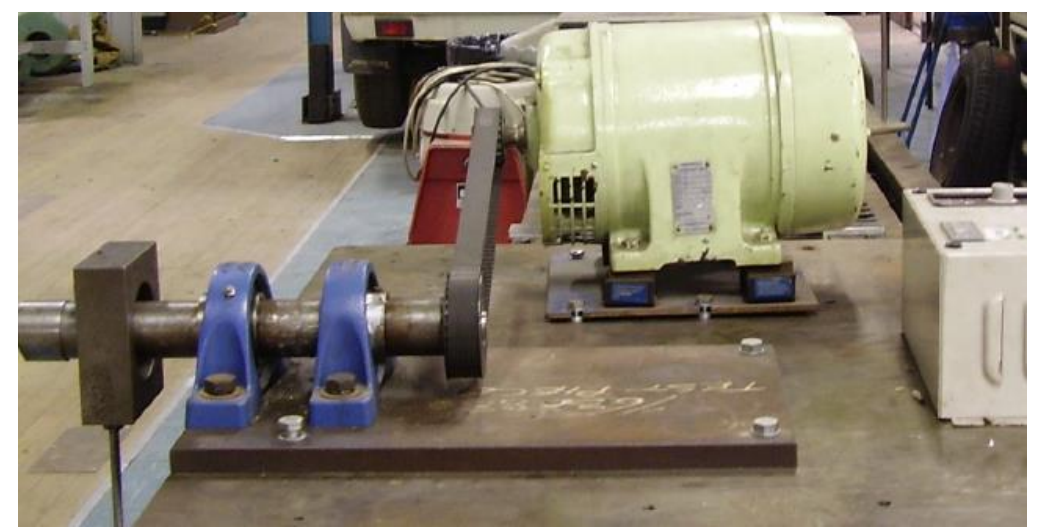

(a)

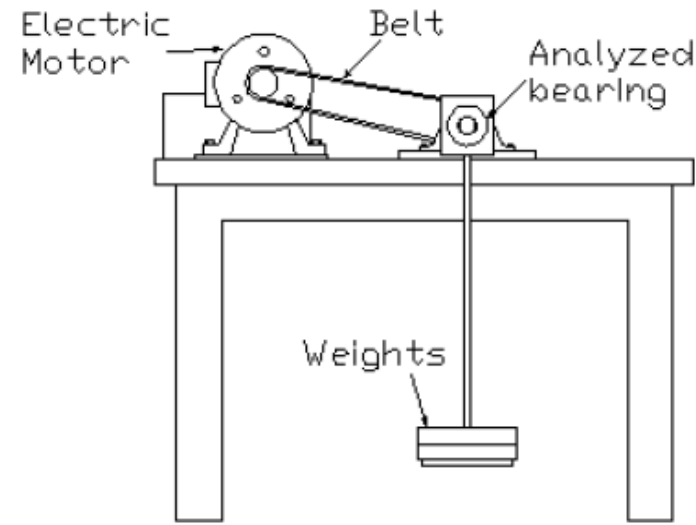

(b)

Figure 4. a,b) The test rig used to collect the signals coming from healthy and faulty bearings. 


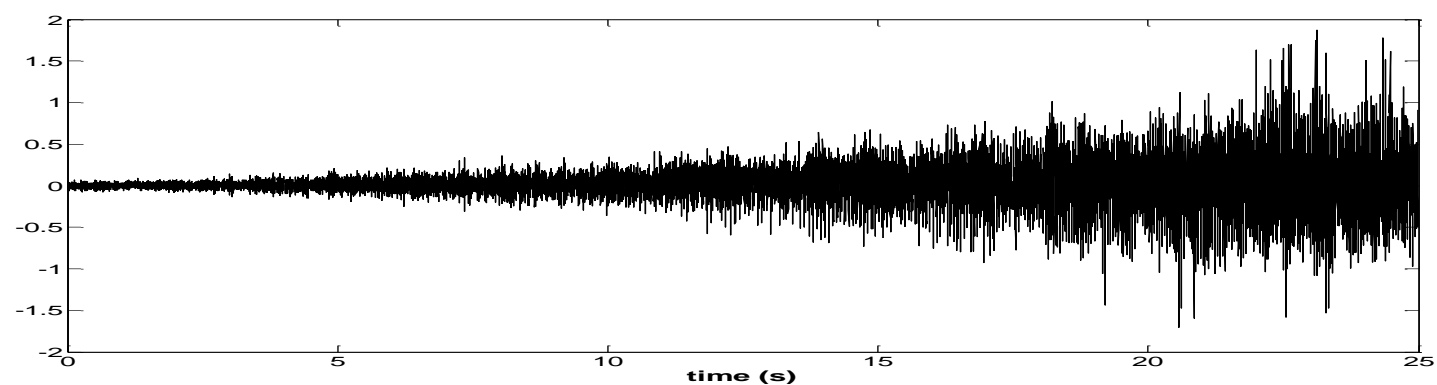

a)

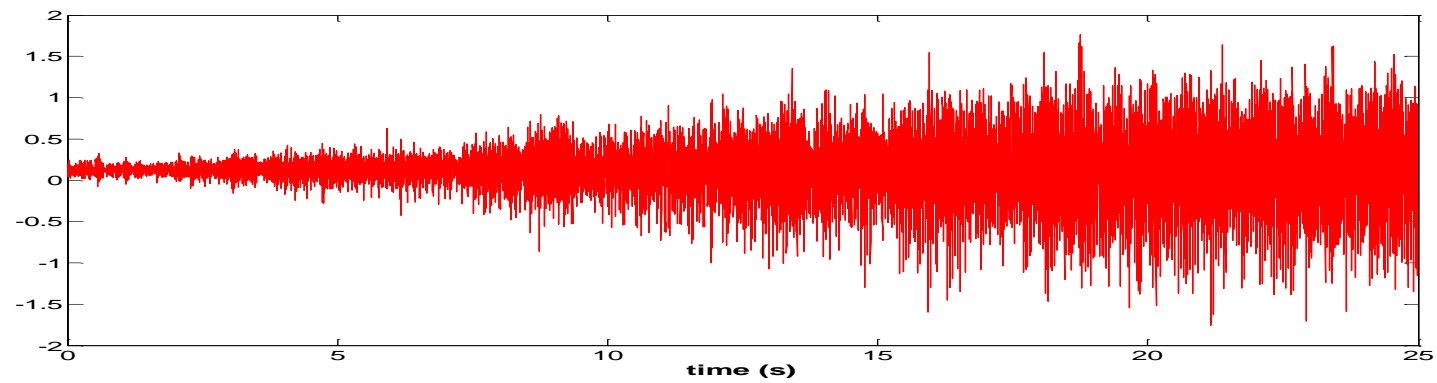

b)

Figure 5. Two acquired signals during run-up condition a) from the healthy bearing b) from the damaged bearing. 

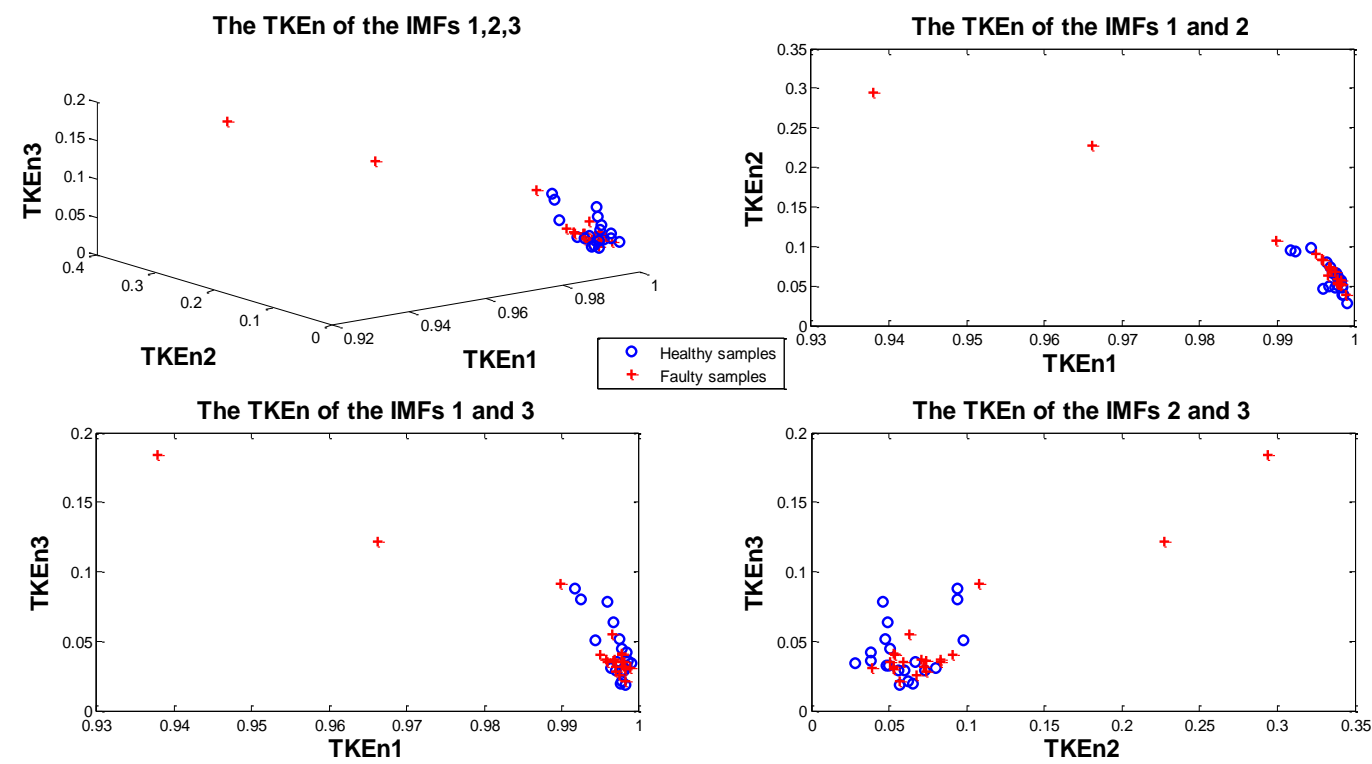

Figure 6. The normalized TKE using the combined method consists of PIEEMD (as the decomposition technique) and TKEO (to create feature vectors). 

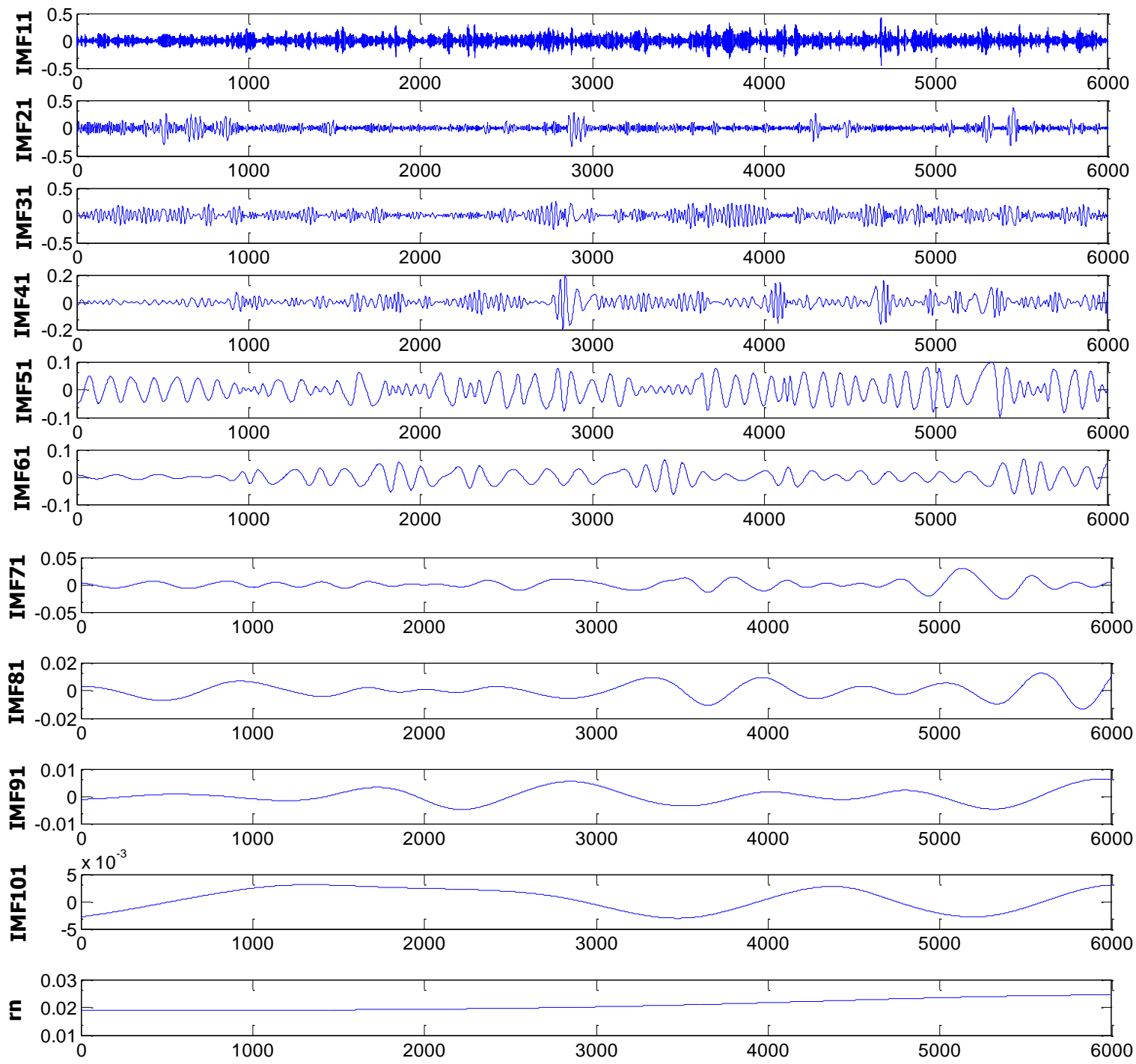

Figure 7. The IMFs of the first segment of the healthy signal (Figure 5(a)) obtained performance improved EEMD (PIEEMD). 


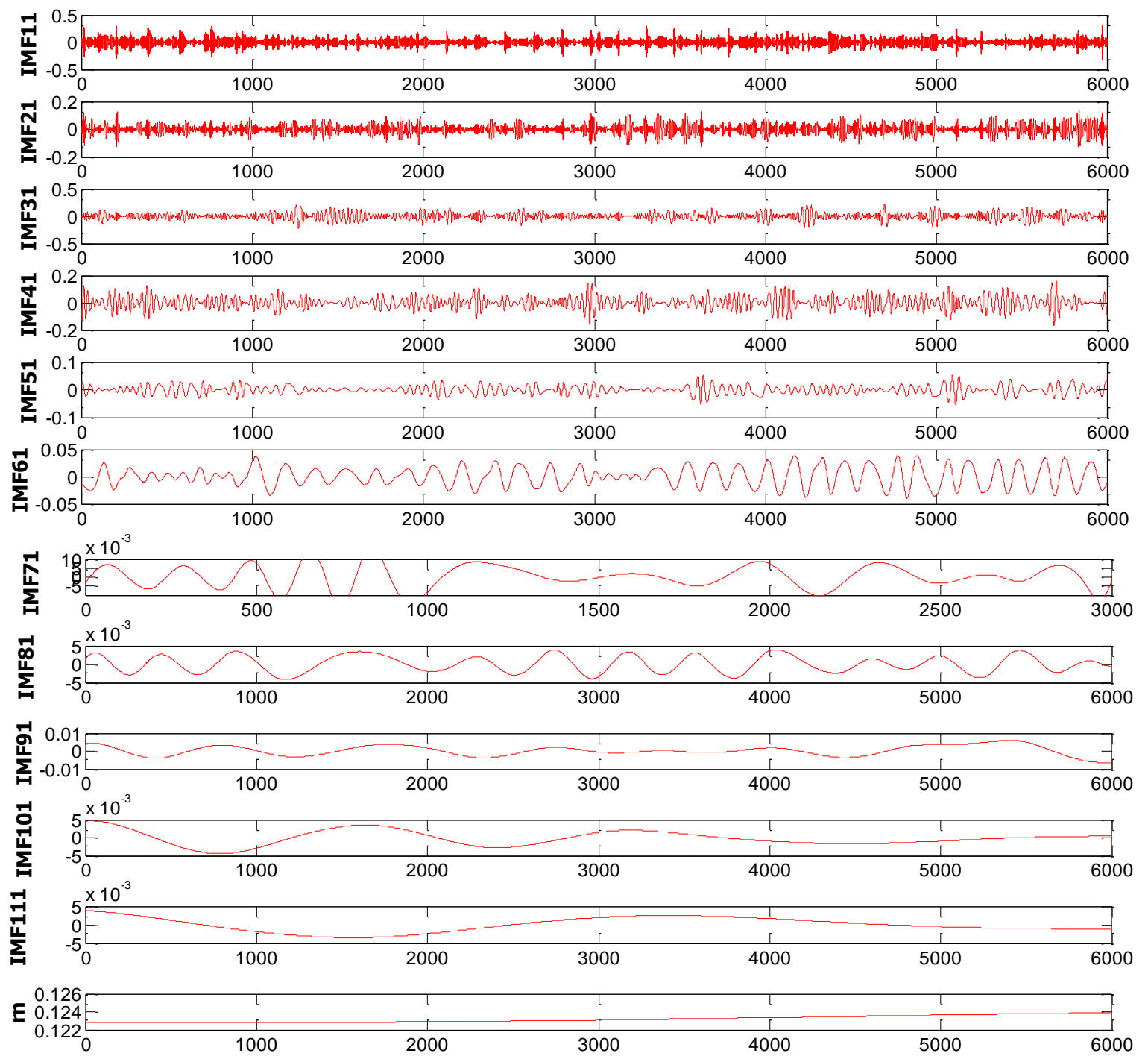

Figure 8 . The IMFs of the first segment of the faulty signal (Figure 5(b)) obtained using performance imperoved EEMD (PIEEMD). 
r1

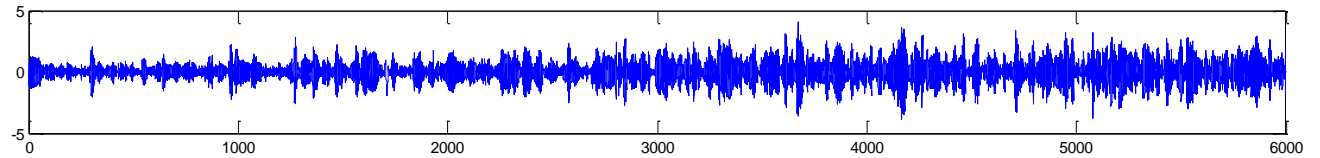

r2

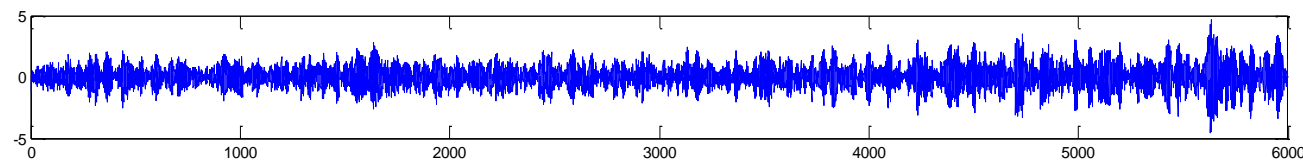

r3

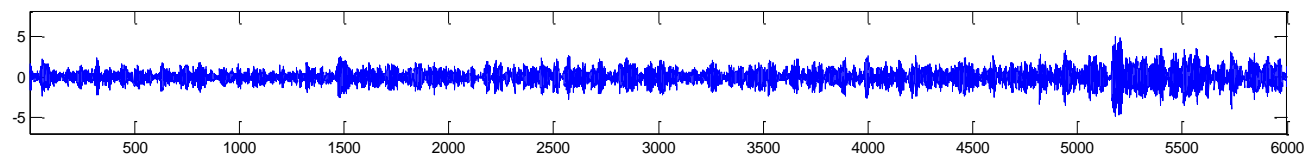

r4

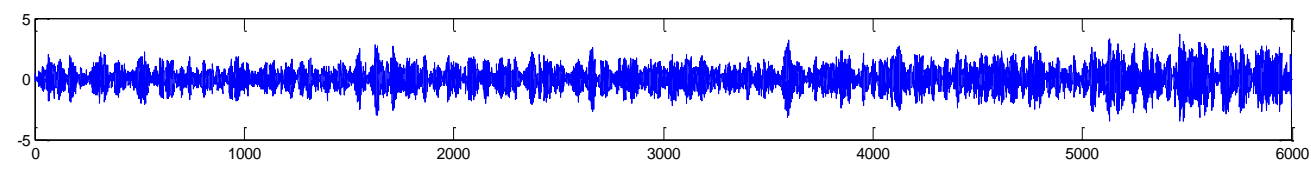

Figure 9. The cointegrating residuals obtaind from first IMFs $\left(I M F_{11} \quad \ldots \quad I M F_{15}\right)$ of the five segments of the signal shown in Figure 5(a). 

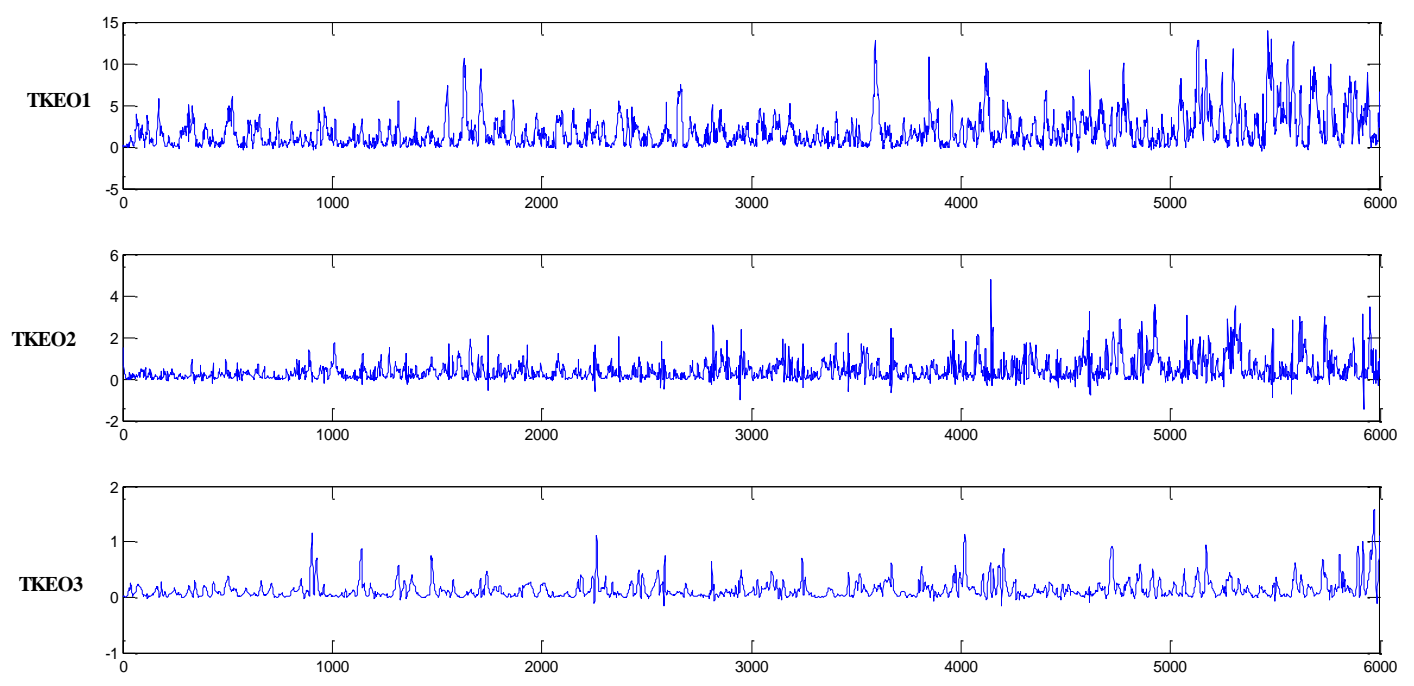

Figure 10. The TKEO applied to the cointegrated residuals obtained from $\left(\begin{array}{lll}I M F_{11} & \ldots & I M F_{15}\end{array}\right)$, $\left(\begin{array}{lllll}I M F_{21} & \ldots & I M F_{25}\end{array}\right)$ and $\left(\begin{array}{lll}I M F_{31} & \ldots & I M F_{35}\end{array}\right)$, respectively. 

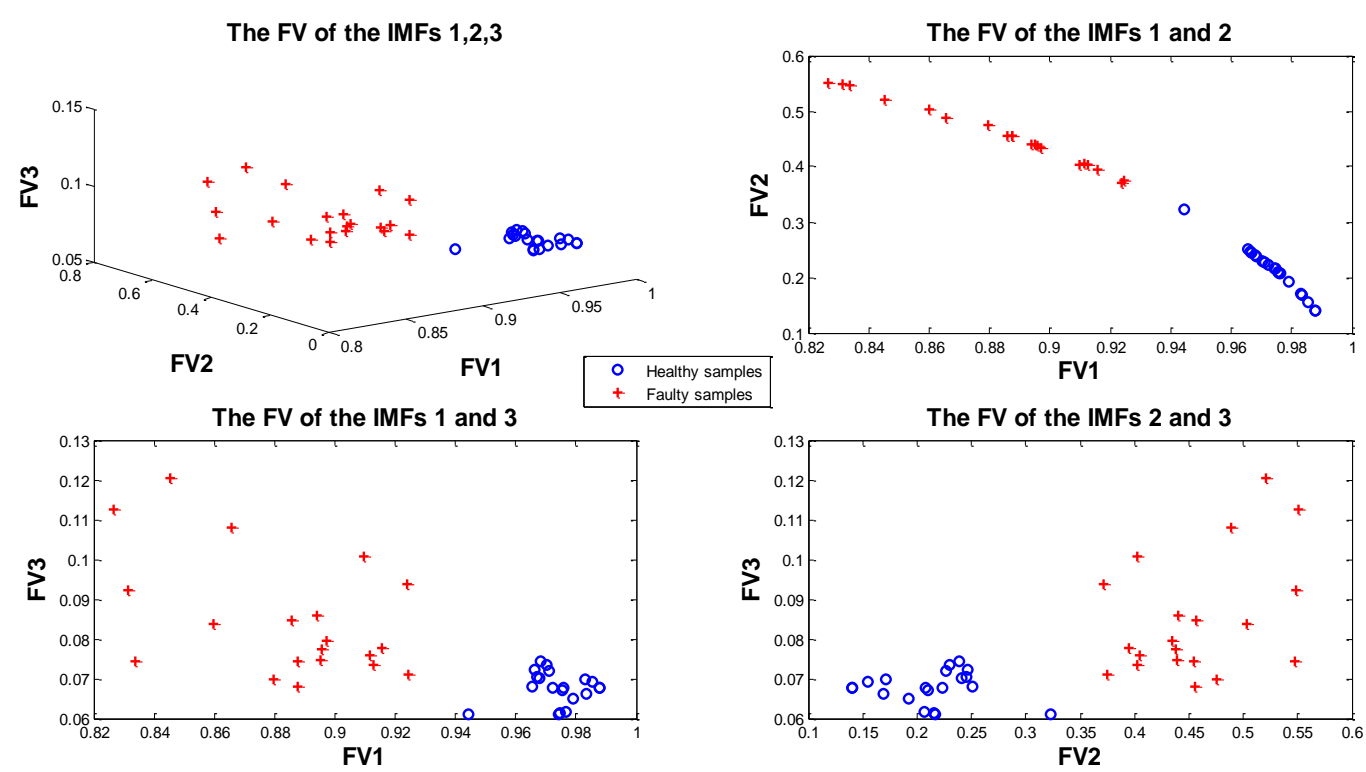

Figure 11. The three dimensional feature vectors obtained from first cointegrated residual. 


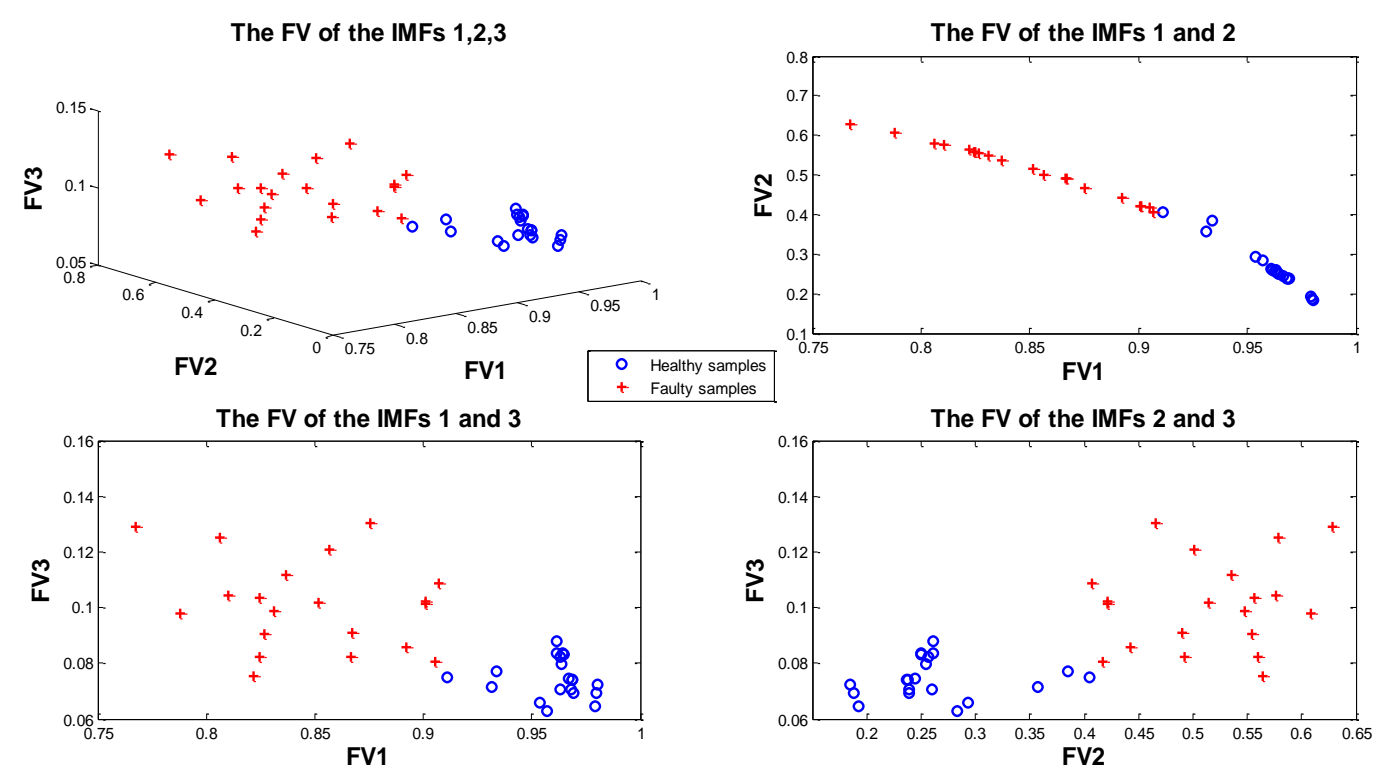

Figure 12. The three dimensional feature vectors obtained from the second cointegrated residual. 

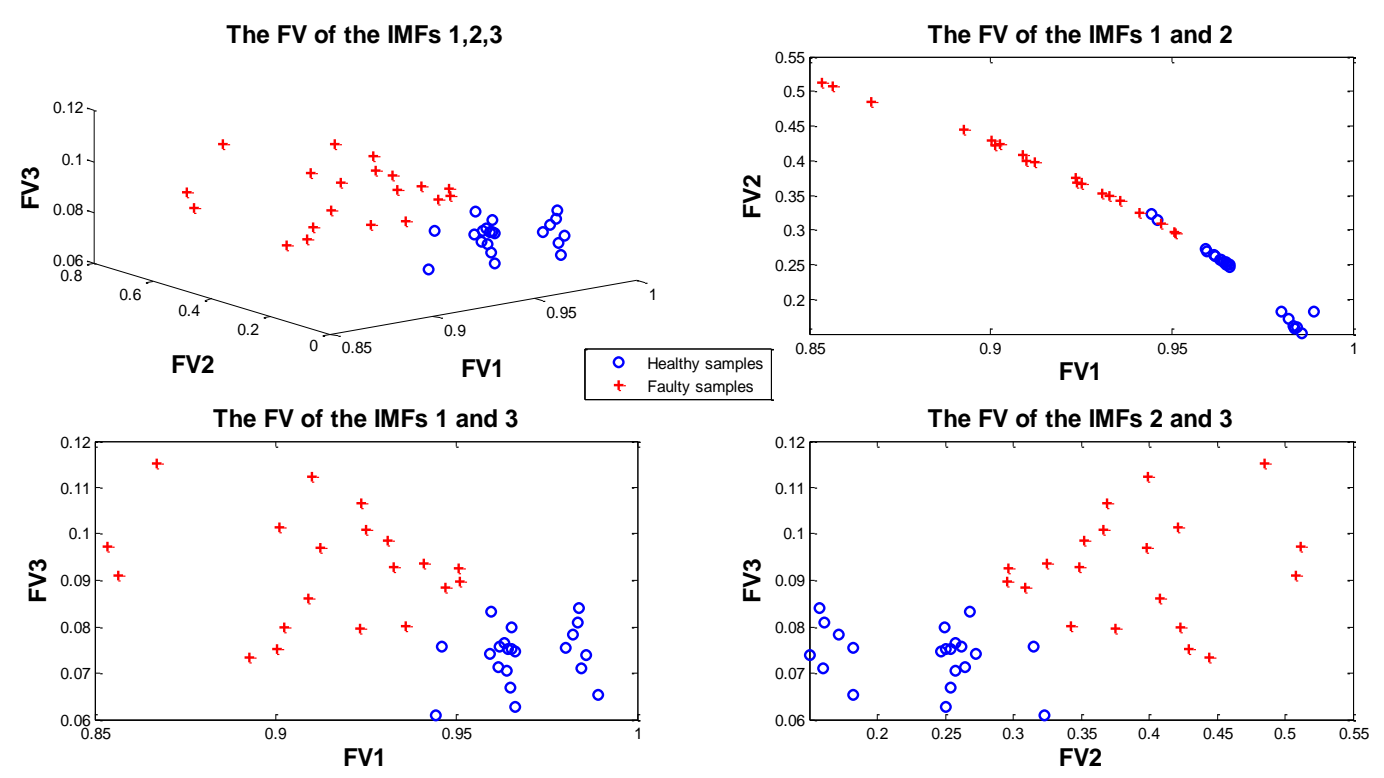

Figure 13. The three dimensional feature vectors obtained from the third cointegrated residual. 

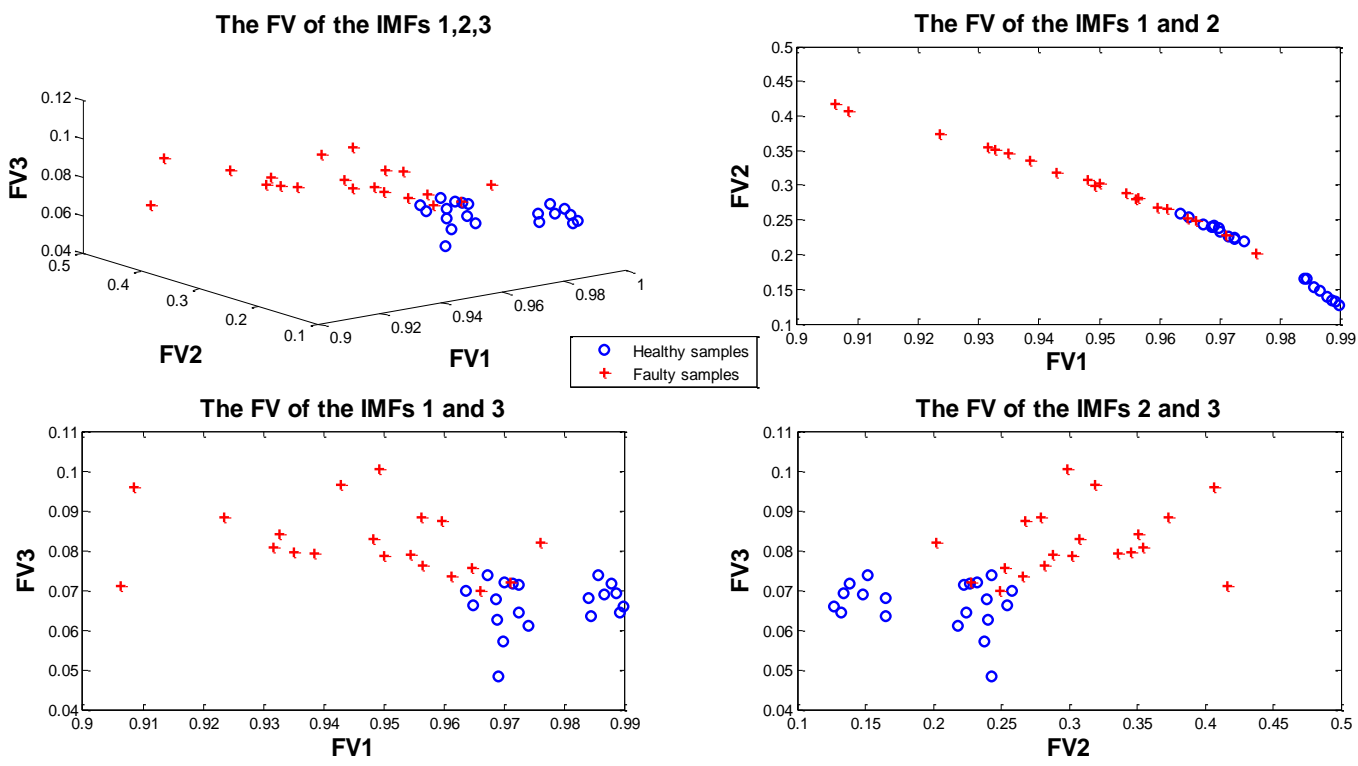

Figure 14. The three dimensional feature vectors obtained from the fourth cointegrated residual. 
Table 1. The results of cointegration for the first IMFs of five segments of the healthy signal shown in Figure 5(a)

\begin{tabular}{llllll}
\hline $\begin{array}{l}\text { Null ranks } \\
\mathrm{r}\end{array}$ & $\begin{array}{l}\text { Null hypothesis } \\
h\end{array}$ & Test statistics & Critical values & $\begin{array}{l}\text { Right-tail } \\
\text { probabilities Prt }\end{array}$ & Eigenvalue $\xi \mathrm{r}$ \\
\hline 0 & 1 & 24466.1675 & 69.8187 & 0.0010 & 0.5753 \\
\hline 1 & 1 & 19328.1531 & 47.8564 & 0.0010 & 0.5604 \\
\hline 2 & 1 & 14397.1423 & 29.7976 & 0.0010 & 0.5589 \\
\hline 3 & 1 & 9486.5483 & 15.4948 & 0.0010 & 0.5565 \\
\hline
\end{tabular}


Table 2. The classification results for different cointegrated residuals

\begin{tabular}{lllll}
\hline $\begin{array}{l}\text { Cointegrating } \\
\text { vectors }\end{array}$ & training & test & Success ratio & Margin \\
\hline 1 & 100 & 100 & $24 / 24$ & 1.00428 \\
\hline 2 & 100 & 100 & $24 / 24$ & 1.00272 \\
\hline 3 & 100 & 91.7 & $22 / 24$ & 0.998041 \\
\hline 4 & 100 & 83.3 & $20 / 24$ & 0.999554 \\
\hline
\end{tabular}

